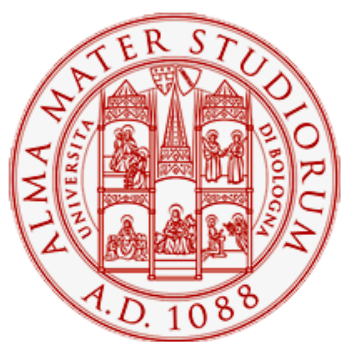

Alma Mater Studiorum - Università di Bologna DEPARTMENT OF ECONOMICS

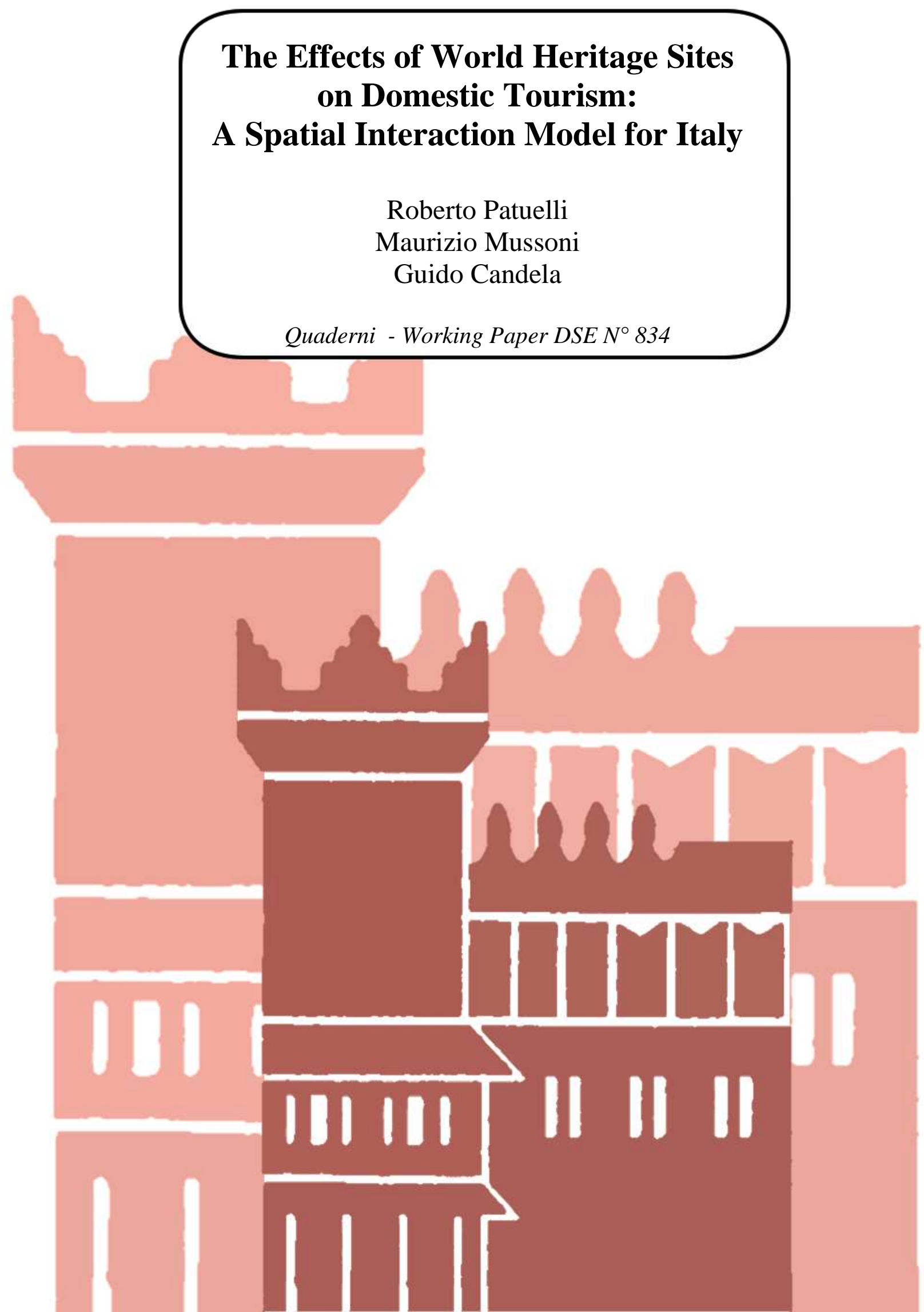




\title{
The Effects of World Heritage Sites on Domestic Tourism: A Spatial Interaction Model for Italy
}

\author{
Roberto Patuelli, Maurizio Mussoni and Guido Candela ${ }^{\bullet \bullet \bullet}$
}

This version: $14 / 06 / 2012$

\begin{abstract}
Culture is gaining increasing importance in the modern tourism industry, and represents a significant force of attraction for tourists (both domestic and international). Cultural tourism allows destinations and regions to expand their customer base, diversify their offer, extend the stay of the tourist, and reduce seasonality. Great efforts are made, by national governments and regions, in order to obtain official designation regarding the relevance of their historical/cultural attractions, for example through UNESCO's World Heritage Sites (WHS) list. Such an aspect seems particularly relevant for a country like Italy, which has a high number of entries in the WHS list, and where regions take an active role in promoting tourism. Using an 11-year panel of domestic tourism flows, we investigate the importance of the regional endowment in terms of WHS from two perspectives: (a) by separately estimating the effects, on tourism flows, of WHS located in the residence region of tourists and in the destination region; and (b) by taking into account potential spatial substitution/complementarity effects between regions due to their WHS endowment. Finally, a sensitivity analysis is offered to evaluate the spatial extent of the latter.
\end{abstract}

Keywords: cultural tourism; domestic tourism; World Heritage Sites; spatial interaction model; Italy; spatial competition.

JEL codes: C23; L83; R12; Z10.

\footnotetext{
- Corresponding author: Department of Economics, University of Bologna, Italy; The Rimini Centre for Economic Analysis (RCEA), Italy. Email: roberto.patuelli@ unibo.it.

• Department of Economics, University of Bologna, Italy; The Rimini Centre for Economic Analysis (RCEA), Italy.

•• Department of Economics, University of Bologna, Italy.
} 


\section{Introduction}

Culture is gaining increasing importance in the modern tourism industry, and represents a significant force of attraction for tourists (both domestic and international). Cultural tourism allows destinations and regions to: i) expand their customer base; ii) diversify their offer; iii) extend the stay of the tourists (overnight stays) and reduce seasonality. For these reasons, national governments and regions make great efforts to obtain official designation for their historical and cultural attractions, for example through the United Nations Educational, Scientific and Cultural Organization (UNESCO) World Heritage Sites (WHS) label.

Such aspect seems particularly relevant for a country like Italy, which is internationally renowned for its abundance of historical and cultural resources, as shown by its high number of entries in the WHS list, and where regions take an active role in promoting tourism. As of 2011, the UNESCO WHS list included 936 sites: 725 were cultural, 183 natural, and 28 mixed, in 153 countries (UNESCO 2011). Italy hosted the greatest number of WHS to date, with 47 sites, corresponding to 5.02 per cent of the total.

Tourism is one of the fastest growing and most profitable sectors of the Italian economy: in 2010, with 43.6 million international tourist arrivals, and international tourism receipts estimated at US\$ 38.8 billion, Italy was the fifth highest tourism earner and the fifth most visited country in the world (UNWTO 2011), behind France, Spain, United States, and China.

In this paper, we analyse Italian 'domestic tourism', which, according to the United Nations, is defined as tourism involving residents of a given country travelling only within the country itself (UNWTO 1994). ${ }^{1}$ Recently, the tourism industry has shifted from the promotion of inbound tourism to the promotion of domestic tourism, because many countries are experiencing increasing competition on the inbound tourism market. Some national policymakers have shifted their priority to the promotion of domestic tourism to contribute to the local economy. Domestic tourism, historically speaking, is in fact the first form of tourism, and today continues to account by far for most of this activity: it is estimated that worldwide, out of the 4.8 billion tourist arrivals per year (2008 figures), 4 billion (83 per cent) correspond to domestic tourism (Pierret 2011). Likewise, UNWTO scholars estimate that, globally, domestic tourism represents:

- 73 per cent of total overnight stays;

- 74 per cent of arrivals and 69 per cent of overnight stays at hotels;

- 89 per cent of arrivals and 75 per cent of overnight stays in other (non-hotel) accommodations.

In Italy, it represents the greatest share of the entire tourism sector, and produces a remarkable macroeconomic impact in terms of value added and labour force. In 2007, domestic tourism provided, on a regional scale, up to 88 and 90 per cent of arrivals and overnight stays, respectively (Massidda and Etzo 2011).

Several studies have investigated whether or not WHS endowment, or more generally cultural offer, increases tourism demand. However, the empirical evidence on this issue is mixed. A number of studies claim that the cultural heritage and attractions of a country are important determinants of tourism demand (see, e.g., Carr 1994; Alzua et al. 1998; Vietze 2008), while others conclude that it is not possible to find a clear positive relationship between cultural endowment and tourism flows (see, e.g., Cuccia and Cellini 2007; Cellini and Cuccia 2009). Regarding specifically WHS endowment, UNESCO declares that obtaining a WHS designation provides significant economic benefits to the host countries (UNESCO 2012). Nevertheless, there is no agreement on this finding

The UNWTO also derived different categories of tourism by combining the three basic forms of tourism: 'internal tourism', which comprises domestic tourism and inbound tourism; 'national tourism', which comprises domestic tourism and outbound tourism; and 'international tourism', which consists of inbound tourism and outbound tourism. 
in the scientific literature, and the debate is still open (see, e.g., Arezki et al. 2009; Yang et al. 2010; Cellini 2011; Yang and Lin 2011).

On the basis of the above discussion, the aim of this paper is to investigate the determinants of Italian domestic tourism flows, with particular reference to the specific contribution of the endowment in WHS. The main concern of this paper is to investigate the importance of the regional endowment in WHS for domestic tourism. We analyse how and to what extent WHS designation affects the flows of tourists between each pair of Italian regions (i.e., between any origin and destination region).

To the best of our knowledge, two specific research questions remain unanswered in the literature on the relationship between WHS endowment and tourism: (1) How differently does the WHS endowment of the origin and destination regions influence tourism flows (i.e., in a push/pull perspective)? (2) Does WHS endowment generate spatial substitution or complementarity between regions?

Accordingly, the objectives of this paper are: (i) to separate the effects on tourism flows of WHS located in the residence region of the tourist (the origin region) and in the destination region; and (ii) to take into account potential spatial substitution or spatial complementarity between regions due to their WHS endowment.

Specifically, our first research question, regarding the origin- and destination-level effects of WHS endowment, can be further stated as follows:

- Does the origin region's WHS endowment push the inhabitants to travel more (or less), influencing tourism outflows (the 'push effect')?

- Does the destination region's WHS endowment attract greater tourism inflows (the 'pull effect')?

In addition to the effect of WHS endowment on inflows and outflows, we are interested in evaluating how the tourists' choices are influenced by the spatial distribution of the WHS. In particular, our second research question can be declined as follows:

- Does the WHS endowment of the regions surrounding the tourist's origin region create a substitution between 'recordable' tourism (hotel arrivals, which involve overnight stays) and daily trips of excursionists (e.g., within the origin region)?

- Does the WHS endowment of the regions surrounding each possible destination region cause spatial competition for tourism demand or spatial complementarity (mutual beneficial effects deriving by trip-chaining) between regions? This question may be particularly relevant from a policy perspective, since regions could use WHS designation for competition or towards joint benefits.

With regard to our second research question, we provide an interpretative framework for the potentially varying effects of WHS endowment of 'neighbouring' destination regions on tourism flows, which is followed by a spatial sensitivity analysis.

To answer the above questions, we set up a spatial interaction model for tourism flows recorded between the 20 Italian regions over the years 1998-2009 (i.e., an 11-year panel). We divide the possible determinants of domestic tourism flows into 'push variables', 'pull variables', and 'deterrence (bilateral) variables', and carry out two Poisson-based (negative binomial) estimations: a two-ways fixed effects model and a spatial filtering-augmented model (including origin and destination fixed effects and a network autocorrelation spatial filter).

The paper is organized as follows. Section 2 briefly reviews the literature on the application of the spatial interaction model in tourism studies. Section 3 briefly presents the literature on the relationship between cultural heritage and tourism, and more specifically between WHS endowment and tourism. Section 4 describes the model and the variables used, the estimation strategy and an interpretative model for the spatial sensitivity analysis offered. Section 5 describes the data set used 
in this study, and presents our empirical findings and their interpretation. Section 6 provides concluding remarks and future research directions.

\section{Modelling Tourism Flows: Spatial Interaction Models}

The spatial interaction model (or gravity model; for an overview, see Haynes and Fotheringham 1984; Sen and Smith 1995), is a modelling framework commonly used in many fields, like commuting, migration, trade, leisure activities, and also tourism. In the case of the latter, it is often used for studying tourism flows between regions or countries (e.g., Uysal and Crompton 1985; Witt and Witt 1995; Khadaroo and Seetanah 2008).

Gravity equations have been estimated since the 1960s for analysing bilateral trade flows (e.g., Tinbergen 1962; Pöyhönen 1963; Anderson 1979; Colwell 1982), and recent applications increasingly emphasize the importance of estimating a theoretically consistent model (e.g., Anderson and van Wincoop 2003; Baier and Bergstrand 2009). The spatial interaction model describes the interaction flow $\left(T_{i j}\right)$ between the origin region $i$ and the destination region $j$ as a function of repulsive forces/push factors at $i\left(R_{i}\right)$ and attractive forces/pull factors at $j\left(A_{j}\right)$, such as the economic size of the origins and destinations, and separation variables referring to the $(i, j)$ pair, such as an inverse function of the friction/distance between the regions $i$ and $j\left(D_{i j}\right)$. A generic formulation of the spatial interaction model can be as follows:

$$
T_{i j}=\frac{f\left(R_{i}, A_{j}\right)}{f\left(D_{i j}\right)} .
$$

Formally, a spatial interaction model is specified as:

$$
T_{i j}=G \frac{R_{i}^{\alpha} A_{j}^{\beta}}{D_{i j}^{\theta}},
$$

where $G$ is a proportionality constant, and $\alpha, \beta$ and $\theta$ are the specific weights of $R_{i}, A_{j}$ and $D_{i j}$ respectively. This multiplicative model is typically estimated after rendering it linear in parameters through log-linearization, or through nonlinear optimization techniques, when constraints are applied in order to respect marginal totals (Wilson 1967, 1970).

Likewise, applications of the spatial interaction model to tourism (see, e.g., Armstrong 1972; Crampon and Tan 1973; Malamud 1973; McAllister and Klett 1976; Swart et al. 1978; Saunders et al. 1981) express bilateral tourism flows $\left(T_{i j}\right)$ as a function of the characteristics of the regions of origin $i$ and destination $j\left(X_{i}\right.$ and $\left.X_{j}\right)$ - factors that augment or distort tourism flows - and of distance, which acts as a proxy for transportation costs. More specifically, in the tourism context, repulsive forces/push factors are associated with leaving $i$ for tourism reasons (tourism outflows), while attractive forces/pull factors are related to going to $j$ for tourism reasons (tourism inflows).

Within this framework, tourism flows (in particular arrivals) can be seen in a similar fashion to migration or commuting flows (e.g., Lowry 1966). Accordingly, tourism flows could be related, for example, to the number of WHS available in the destination, and to other control variables evaluated at the same location, like crime indices and other cultural proxies. On the other hand, flows could also be dependent on the number of WHS available in the origin, as well as on the population basin or per capita income, and finally on the distance between the origin and the destination. An alternative interpretation of the spatial interaction model for tourism consists of applying a 'commodity version' of the model, according to which tourism is essentially seen as a form of international trade, and tourism flows are treated as a traded good (Leamer and Levinsohn 1995; Eilat and Einav 2004; Yang et al. 2010). 
In the empirical literature on international and domestic tourism (e.g., Sheldon and Var 1985; Calantone et al. 1987; Kim and Fesenmaier 1990; Gardini 1998; Zhang and Jensen 2007; Nicolau 2008; Keum 2010; de la Mata and Llano-Verduras 2011), the most frequently used dependent variables have been tourist arrivals or overnight stays, as well as tourist expenditures or receipts. Regarding the explanatory variables, there is undoubtedly a wide range of possible determinants of tourism demand, the most prominent being income (GPD at the macro level), relative tourism prices, transportation costs, exchange rates, and qualitative factors in destination regions.

In particular, the level of income of the potential tourist affects his/her ability to pay for travel (Sheldon and Var 1985), while GDP (observed both at the origin and at the destination) is used as a proxy for market size, and represents push and pull factors that influence the value of tourism flows. $^{2}$ Other important determinants of tourism demand are: the relative prices of goods and services purchased by tourists in the destination, compared with the origin and the competing destinations (see, e.g., Gerakis 1965; Edwards 1976; Bond et al. 1977); transportation costs (usually proxied for by distance), which refer to the cost of round-trip travel between the origin and destination regions; the exchange rates between the currencies of origin and destination (mostly relevant in the case of international tourism). Demand for travel to a particular destination is expected to be positively related to both income in the origin and exchange rates, and negatively related to both transportation costs and relative tourism prices.

Further explanatory variables included in tourism demand models (for an overview, see Sheldon and Var 1985; Lim 1997) are: trip motive or frequency; destination attractiveness and endowment (climate and temperature, natural environment, pollution and environmental quality, culture and history, cultural heritage, WHS); political, social, cultural and sporting events in the destination; destination marketing or promotional expenditures (information, tourist services, public expenditure for culture activities and events, etc.); supply/capacity constraints of tourist accommodations (carrying capacity); supply-side variables, like tourism and transport infrastructure of the destination; social variables capturing the role of public safety, such as the diffusion of small and/or violent crime (Eilat and Einav 2004; Massidda and Etzo 2011); a time trend variable capturing long-run change in tourist tastes (Barry and O'Hagan 1972) or the steady change in the tourist mix (Fujii et al. 1985, 1987); lagged variables accounting for dynamics, such as the previous values of income, relative prices, exchange rates, and foreign investment; proxies for business travels, such as trade, foreign direct investment, or capital outflows. Finally, a large number of qualitative factors (typically accommodated by means of dummy variables) may influence the decision to demand tourism, including the tourists' attributes (gender, age, education level, and employment/profession), which may affect leisure time availability or similar constraints.

In this paper, we choose as a dependent variable the bilateral tourism flows (in terms of arrivals) between each pair of Italian regions, while, in terms of explanatory variables we consider the main determinants outlined above. Our variable of interest is the number of WHS existing in each Italian region. Finally, on the basis of the preceding discussion, we argue that the spatial interaction model is a suitable tool for investigating the research questions proposed in this paper. We build our model starting from a standard spatial interaction model for tourism, and we subsequently augment it by including key variables related to WHS.

To further investigate our research questions, the next section briefly presents the literature and the ongoing debate on the relationship between cultural heritage and tourism, and more specifically between WHS endowment and tourism.

2 Further specifications in the literature have used population (Linnemann 1966) in order to capture size effects. 


\section{Cultural Heritage, WHS Endowment and Tourism: The Evidence}

\subsection{Cultural Heritage and Tourism}

Many studies aim to investigate whether the cultural endowment and heritage of a country can be considered as an important determinant of tourism demand, for either domestic or international tourism. Several studies claim that cultural heritage and attractions, in many developed countries, are becoming a major driving force for further growth of the tourism market, and that the abundance and diversity of cultural resources are essential tourism assets for a country to develop its tourism industry (see, e.g., Carr 1994; Markwell et al. 1997; Alzua et al. 1998; McIntosh and Prentice 1999; Herbert 2001; Vietze 2008). According to these studies, all combinations of natural, cultural, and manmade elements are closely related to the demand for tourism, since they are unique to the single tourism destinations and cannot be transferred or reproduced in other locations (Dritsakis 2004). Consequently, a location endowed with natural landscapes, historical sites, cultural traditions, and heritage could have a competitive advantage when it comes to attracting tourists. Moreover, from the viewpoint of domestic tourism, heritage tourism is recognized as an effective way of achieving the educational function of tourism (Light 2000; Dean et al. 2002).

However, other studies stress that cultural sites and attractions are not effective in attracting tourism flows (see, e.g., Cuccia and Cellini 2007). Cellini and Cuccia (2009) find evidence that tourism flows Granger-cause cultural sites attendance, while the reverse does not hold, that is, a unidirectional long-run causal link emerges, but running from tourism flows to cultural sites attendance. Consequently, it would not be possible to sustain the hypothesis that cultural attractions can promote tourism in the long run, at least at the aggregate level, and, at most, the role of cultural sites would be limited to being a marginal product within a destination's tourism basket or a possible solution towards decreasing seasonality. Moreover, contrasting evidence on the relationship between attendance of cultural attractions and tourism flows was found for other 'cultural goods' as well, such as temporary arts exhibitions (Di Lascio et al. 2011) or museums and monuments (Cellini and Cuccia 2009).

\subsection{WHS Endowment and Tourism}

We focus on the effects of UNESCO's WHS designation on Italian domestic tourism flows, rather than on the overall effects of 'cultural heritage', ${ }^{3}$ or of generic cultural sites and attractions. According to UNESCO, there are significant economic benefits to obtaining a WHS designation. This is due to an 'increase in public awareness of the site and of its outstanding values', which would in turn spark an increase in tourist activities and visitation to the area, with related economic benefits not only for the destinations hosting the cultural and natural sites, but also for the local economy (UNESCO 2012).

There is a large body of literature that investigates the impact of WHS endowment on tourism, although no final evidence appears to have been reached. The literature on this topic can be divided into four main streams, depending on the conclusions on the impact of WHS endowment on tourism: (i) the literature which generally suggests a positive effect; (ii) the empirical studies which claim that WHS designation has a positive but relatively small effect; (iii) the recent studies which find an insignificant effect for tourism but an important effect in terms of protection of heritage; and (iv) the literature on the overall negative aspects of WHS designation.

The early literature focuses mainly on the benefits of WHS designation. Its primary motivation was the protection and preservation of outstanding natural and cultural sites, but since the mid 1990s the literature began to analyse also its potential socio-economic benefits, mostly in terms of

3 'Cultural heritage' is defined in Article 1 of the Convention concerning the Protection of the World Cultural and Natural Heritage (adopted by UNESCO in 1972) as monuments, groups of buildings and sites that are of 'outstanding universal value from the point of view of history, art or science' and form the 'aesthetic, ethnological or anthropological point of view'. 
possible increases of tourism flows and revenues (see, e.g., Ashworth and Tunbridge 1990; Drost 1996; Pocock 1997; Shackley 1998; Thorsell and Sigaty 2001). The main conclusions were generally that WHS designation increases the popularity of a location, acts as a 'magnet for visitors', and is 'virtually a guarantee that visitor numbers will increase' (Shackley 1998, Preface). Therefore, according to this strain of the literature, WHS designation helps building a 'destination image'. Moreover, according to more recent studies (Arezki et al. 2009; Yang et al. 2010; Yang and Lin 2011), WHS are increasingly becoming one of the main touristic resources in many countries. The UNESCO WHS label would provide a surplus value to the sites, with respect to the generic cultural, historical and natural sites of a country, as it is expected to have a (strong) impact on tourism demand, and therefore on tourist arrivals, revenues and jobs creation, all important aspects for regional development. For example, WHS are nowadays widely used in marketing campaigns to promote tourism, to increase the visibility of destinations.

A second stream of (empirical) literature focuses on the quantification of the impacts of WHS designation on tourism flows and revenues. These studies provide mixed results, and generally suggest that WHS designation has a positive but relatively small impact on tourism flows (see, e.g., Buckley 2004; van der Aa 2005; Blacik 2007; Soares et al. 2007; Bové Sans and Laguado Ramírez 2011; VanBlarcom and Kayahan 2011). These studies find a positive association between WHS designation and tourism flows, but in some cases the evidence presented is not conclusive. Di Giovine (2009) argues that WHS designations are not 'impotent political performances that lead to the commercialization of local monuments', but instead are the building blocks of a new social and economic system. Other studies analyse the relationship between WHS endowment and tourism for specific countries; for example, Buckley (2004) for Australia, Blacik (2007) for Africa, Soares et al. (2007) for Portugal, VanBlarcom and Kayahan (2011) for Canada, and Bové and Laguado (2011) for Spain. Most of the sites reported an average increase of 1-5 per cent per year in tourists since the designation. However, the causal link between WHS designation and increased tourism flows above existing tourism trends is found to be relatively weak, particularly for sites that were already major attractions prior to their designation. In fact, according to VanBlarcom and Kayahan (2011), sites that are well known globally appear to benefit less from WHS designation relative to sites with a lower global profile. Furthermore, Bové and Laguado Ramirez (2011) claim that, in order to exploit a WHS for tourism, it is necessary to enforce policies of external promotion and communication, in order to clearly position the destination within the tourism market as a 'cultural heritage destination'. Finally, according to van der Aa (2005), WHS status leads in particular to an increase in the number of international tourists, who tend to stay longer and spend more than domestic tourists.

A third and more recent stream of literature finds an insignificant impact of WHS designation in terms of tourism flows, but an important effect in terms of heritage protection (see, e.g., Hall and Piggin 2001; Hall 2006; Cellini 2011). Cellini (2011) claims that the effects of the WHS designation on tourism demand are far from clear-cut and robust. As a consequence, the main motivation for WHS recognition would only be a better protection of heritage, through the availability of additional funds. Hall (2006) states that the common perception is that WHS designation leads to increased commitment and tourism flows, and to increased public support for site maintenance and preservation. However, he notes that there are actually many other implications of a WHS designation, including 'potential changed access and use of the site and related environmental issues, new regulatory structures and altered economic flows'. The author concludes that much attention has been given to WHS designation, rather than to how effectively the designation has been implemented.

Finally, a fourth stream of literature suggests an overall negative impact of WHS designation (see, e.g., Mossetto 1994; Gamboni 2001; Meskell 2002; Frey and Steiner 2011). In particular, according to some studies ( $\mathrm{Li}$ et al. 2008; Yang et al. 2010), WHS designation might have a negative impact on heritage conservation, since the sites could attract an excessive number of visitors, carrying the danger of seriously compromising the environmental and cultural integrity of the sites. 
An alternative stream of literature focuses on the costs of WHS designation, in a comparison with the related benefits, and conducts cost-benefit analyses (CBA). PriceWaterhouseCoopers LLP (2007) carries out a CBA of WHS designation in the UK, and finds an increase in tourism flows by 0-3 per cent, compared to an increase in costs around $£ 500 \mathrm{~K}$, including bidding costs, cost of the management plan and management costs of the WHS. Research Consulting Ltd and Trends Business Research Ltd (2009) report that approximately 70-80 per cent of WHS sites appear to be doing little or nothing to exploit the WHS designation towards significant socio-economic impacts. The authors conclude that management organization, marketing promotion and stakeholders' perception of WHS status matter. They argue that the small-to-null economic impacts of WHS designation found in the early literature are not surprising, since most of the sites analysed lack the motivation to promote their WHS designation in order to generate economic gains. VanBlarcom and Kayahan (2011) find evidence consistent with the conclusions of Research Consulting Ltd and Trends Business Research Ltd (2009): the economic impact of the WHS is site-specific, and is subject to overall tourism trends affecting the level of tourism flows. In other words, WHS designation alone is not sufficient to stimulate transformational change, so the local policymakers must enforce policies aimed at capitalizing upon it, and invest in the other links within the tourism chain to gain benefits through a 'ripple' effect.

On the basis of the above discussion, we believe that it is highly relevant to further investigate and assess the extent to which WHS attract tourists, in order to gather information that can be critical towards implementing effective tourism policies, in terms of both promoting cultural tourism and managing potential damages caused by the overloading of tourists. In particular, we aim to shed light on the role of WHS endowment in trip generation and assignment, that is, on its influence over the outflows and inflows of tourists. The studies reviewed above investigate the impact of WHS endowment on tourism by applying a variety of econometric models. However, none of them faces the problem from a spatial interaction perspective. In addition, the current applied literature does not provide empirical evidence on how the spatial distribution of amenities (in our case, WHS) affects tourists' trips, in a competing destinations (Fotheringham 1983) or tripchaining perspective. Following these reflections, the subsequent section outlines the empirical model used in this paper, and further specifies our research questions and their operationalization.

\section{Model and Estimation Strategy}

\subsection{Model}

Most applications of the spatial interaction model in the tourism domain regard international tourism. Nevertheless, models for international or domestic tourism do not differ in their fundamentals, but with respect to the set of explanatory variables considered. In the international domain, exchange rates, institutional factors, trade intensity, and common characteristics of countries (such as language) are important drivers of touristic flows. For domestic tourism, such variables are generally not relevant (institutions and language tend to be invariant within a country, and interregional trade is seldom measured) or indirectly related (e.g., the substitution effects generated by exchange rate variations may alter the distribution of domestic tourism). On the other hand, variables relating to demand (e.g., GDP or per capita GDP) or supply (e.g., kms of coastline, investment in recreational activities, cultural offer) factors can easily be interpreted in a domestic setting as well.

We start from a standard spatial interaction model, by considering two types of variables: originrelated and destination-related. In addition, bilateral variables are frequently given in the context of international tourism, while geographical distance remains a variable of interest in the domestic context as well. In particular, although most origin or destination variables can be reformulated (and reinterpreted) in a bilateral fashion (i.e., in terms of differentials), in our modelling framework we prefer to maintain the bidimensionality of our information, so to differentiate the effect of the 
characteristics of the origins on outgoing flows, and of destination characteristics on incoming flows.

We model interregional tourism flows, measured as arrivals in hotels and other accommodation outlets, as a function of a number of control variables incorporating push and pull factors, including regional population and GPD, evaluated at both the origin and destination regions, in order to capture information on market size and income (i.e., conditional to market size), respectively. For the origin region, these variables are commonly expected to be associated with a positive effect on tourism flows. For the destination region, GDP can still be interpreted in a market size fashion, to account for the share of business trips over total flows, and both GPD and population may have an influence on the choice of destination both as a positive effect, proxying for the level of economic development, and as a negative effect, since tourists could prefer visiting less-industrialized (or less dense) and more relaxing areas (see, e.g., the snob effect, in Candela and Figini 2012). Because income tends to influence consumption choices with a delay, we use lagged GDP.

Furthermore, we control for the level of prices in the origin and destination regions, to cope with differences in the costs of living. More precisely, we use a price index computed regionally and specifically for the hotels and restoration sector. Destination prices are commonly used in the tourism modelling literature and are expected to negatively affect inflows, while origin prices may be expected to have the opposite effect, pushing tourists out in search of price-effectiveness. In other applications, the ratio between destination and origin prices is used to permit substitution between the choice of a destination and the local tourism/stay-home hypothesis (or, in the international tourism framework, between foreign destination and domestic tourism; Witt and Witt 1995).

We include in the model further regional characteristics, aiming to account for the diffusion of crime, public spending in recreational activities, regional reliance on the tourism industry and seasonal concentration of tourism, public transport efficiency, cultural demand, and environmental quality. In detail, with regard to crime diffusion, we employ two indices, which denote small crime and violent crime, respectively. With regard to the destination, regions with high crime rates may be expected to show a diminished interest from tourists, all being equal, because of safety concerns. On the other hand, a region with renowned tourism sites may actually attract further potential criminals seeking potential victims (Eilat and Einav 2004; Dhariwal 2005), therefore incorporating the medium-long run level of local tourism demand. As far as the origins are concerned, we may expect residents of high-crime regions to be more likely to travel, in order to alleviate, at least temporarily, their risky condition. However, this effect may indeed be difficult to catch, even conditionally to per capita income, if the income distribution is strongly unequal (that is, a vast share of the population would not be able to afford travelling). Finally, to control for possible endogeneity of the tourism-crime relationship, we enter the small crime and violent crime variables in the model in lagged form.

In order to account for the different tourism 'vocation' of regions, and their reliance on this sector, we include a variable reporting the share of regional value added of the macro-sector including commerce, hotels and restaurants, transports and communications over total value added. Similarly, we account for the share of regional public spending invested in recreational, cultural and religious activities. A third variable accounts for the regions' reliance on off-season tourism.

We may expect the tourism specialization variable to account, for destination regions, for most of past unobservable factors that make a region a staple in (domestic) tourism, and therefore to be positively correlated with flows. With regard to origin regions, sign expectation is ambiguous. On the one hand, residents of tourism-relying regions might tend to have repulsion for traditional (hotel) tourism. On the other hand, a phenomenon of tourism 'addiction' a la Becker (1996) might be observed, for which the residents of such regions would appear to travel more, on average. Public spending in recreational/cultural activities represents, in our model, the investment of local administrations towards attracting tourists. As such, we should expect a positive effect on flows with regard to destination regions. However, this spending can also be seen as the administrations attempt to recuperate a medium-term scarcity in tourism demand, eventually showing a possible 
negative correlation with tourism flows. A similar reasoning goes for the origin region, where the residents may be more likely to stay or to undertake shorter (one-day) trips, if local recreational and cultural activities generate a significant interest, while if spending efforts are made in order to catch up with more successful regions, we might observe greater tourism outflows. Finally, the variable for the number of off-season tourists (per inhabitant) accounts for the regions' success in extending their period of touristic consumption, for example by diversifying their touristic offer. Regions with higher off-season tourism are expected to experience greater inflows, while a sign expectation at the origin level can hardly be formulated. For both tourism specialization and recreational spending, we include the variables in lagged form, to allow for habit formation and the fact that, for example, for public events to develop a 'reputation', longer periods of time are needed.

On the supply side, more variables are included, namely the share of satisfied customers of the regional railway service, and the percentage of non-bathable coastline. The former accounts for the provision and quality of transport infrastructure in the regions, which can be expected to influence flows both at the origin and at the destination. The latter is an indicator of the quality of waters for coastal regions (in Italy, 15 of 20 regions have access to the sea), and therefore should be expected to negatively influence flows to the destination region, and positively influence outflows from the origin region.

On the demand side, we account for the quality of the cultural offer by including the average number of visitors per state museums, and the number of tickets sold per inhabitant for theatrical and musical events. Both variables can be expected to have a positive effect on inflows of tourists, while the expected sign at the origin is unclear: on the one hand, higher quality attractions in the region of residence may diminish outflows; on the other hand, we might again observe a phenomenon of 'addiction', for which the residents of a cultural endowed region might travel more to experience further cultural goods.

The first research question we aim to answer is whether the regional endowment in WHS has a measurable effect on domestic tourism flows, and how this (potential) effect can be decomposed in an origin-level effect and a destination-level effect. More precisely, we aim to evaluate whether WHS-endowed regions (1) generate more or less recordable outflows, and (2) attract greater inflows.

With respect to the first case, both a positive and a negative effect may be expected. On the one hand, we might expect regions which are endowed in WHS to experience lesser tourist outflows, if the residents' opportunity cost linked to travelling is evaluated on the basis of the lower opportunity cost of visiting local valuable cultural sites. As a result, if potential tourists prefer to travel locally, in particular by daily excursions, recorded flows - which are collected at hotels and other accommodations - would be diminished, leading to a negative push effect. On the other hand, a positive push effect might be found if the region's residents tend to be more curious, and therefore to generally travel more, when they are locally surrounded by cultural sites (because of love for variety). The second case is more straightforward, that is, WHS endowment allowing regions to attract a greater number of tourists. We expect a positive sign for this effect, since a negative one could only be justified by a crowding out effect of international tourists (not considered here) on domestic tourists.

The paper's second research question deals with the tourists' behaviour with respect to the spatial distribution of the WHS endowment of the regions. As above, we can subdivide it in two subquestions: (1) Does the WHS endowment of the regions surrounding the origin influence tourists outflows? (2) Does the WHS endowment of the regions surrounding a destination influence its inflows?

The first subquestion can be reconducted to the justification of the similar question we introduced above with respect to the WHS endowment of the origin region. We hypothesize that, the higher a WHS endowment is available in nearby regions, the more potential tourists could be induced to substitute 'traditional' tourism (i.e., hotel arrivals, involving overnight stays, and therefore recordable) with 'daily excursions', inducing a negative effect on recorded outflows. The second subquestion has both an empirical interest and a policy one. Fotheringham (1983) has 
shown, in his work on competing destinations theory, that the spatial interaction model is better specified when the clustering of possible destinations is explicitly taken into account within the theoretical model leading to a multinomial logit (at the individual level). In other words, he showed that the individual does not have perfect information on the characteristics of all destinations, and that he/she will consider, for each possible destination, alternatives clustered in its proximity. Eventually, this boils down to incorporating in the spatial interaction model an additional variable describing the alternative destinations, usually in terms of accessibility. In tourism modelling, an attempt to include such aspects in an empirical model is made by Khadaroo and Seetanah (2008), who, in a study on international tourism, include a binary variable for the presence of nearby alternative destinations.

With regard to our case study, we model accessibility to alternative destinations by considering the WHS endowment of the regions surrounding each destination (i.e., we use a rook contiguity definition of proximity ${ }^{4}$ ). We hypothesize that the tourist's set of information - for the purposes of evaluating a destination's attractiveness - is limited to just the destination set of all neighbouring alternative destinations. We may frame this approach within the more general framework of the prominence models described in Sen and Smith (1995), which includes, among others, Fotheringham's model of competing destinations (Fotheringham 1983). An estimated positive effect for the endowment of neighbouring destinations would therefore imply that a phenomenon of trip-chaining exists (spatial complementarity), in which the tourists consider potential visits to WHS outside of the destination region (but relatively close). On the other hand, a negative sign would instead imply that the 'competition' of alternative WHS decreases a region's inflows (spatial competition). This aspect assumes great relevance from a policy perspective, in a framework like the one of Italy, where regional agencies are in charge of promoting tourism, and where lobbying activities for the designation of additional WHS is strong.

The two research questions outlined above are operationalized in a spatial interaction model by including, for the first research question, two variables, $W H S_{i}$ and $W H S_{j}$, accounting for the WHS endowment of each origin and destination region, respectively. With regard to the second question, we include the average WHS endowment of regions contiguous to each origin and destination. The new variables, $L . W H S_{i}$ and $L . W H S_{j}$, are computed as $\mathbf{W} * W H S_{i}$ and $\mathbf{W} * W H S_{j}$, respectively, where $\mathbf{W}$ is a $20 \times 20$ row-standardized spatial weights matrix defining contiguity relations of proximity between all regions. The resulting augmented model can then be written as follows:

$$
T_{i j}=f\left(X_{i}, W H S_{i}, L \cdot W H S_{i}, X_{j}, W H S_{j}, L \cdot W H S_{j}, D_{i j}\right)
$$

where $T_{i j}$ is the flow of tourists from region $i$ to region $j, X_{i}$ and $X_{j}$ are the vectors of values for the origin (push) and destination (pull) variables given above, respectively, and $D_{i j}$ is the geographical distance between the two regions. Because of data availability, most variables are lagged, in the empirical specification, by one or two years. By means of Equation (3), we can separate the main effect (direct effect) of WHS endowment of the origin and destination on tourism flows $\left(W H S_{i}\right.$ and $\left.W H S_{j}\right)$ from the indirect effect of WHS endowment of their surrounding regions $\left(L . W H S_{i}\right.$ and L. WHS $S_{j}$.

The following sections describe the empirical estimation method and provide an interpretative framework for the varying direct and indirect effects of WHS endowment on tourism flows, according to a spatial sensitivity analysis.

4 When a contiguity rule is applied to define proximity, two regions are defined as neighbours if they share a border. In rook contiguity, the common border has to have length greater than zero, while in queen contiguity common borders of length zero are allowed as well. 


\subsection{Estimation}

We estimate our model for a panel of all 20 Italian regions, and 12 years (1998-2009). Considering the time dimension, we can again generically write Equation (3) for estimation purposes, as follows:

$$
T_{i j t}=f\left(\alpha_{i j}, \text { year }, X_{i t}, W H S_{i t}, L . W H S_{i t}, X_{j t}, W H S_{j t}, L . W H S_{j t}\right) \text {, }
$$

where $\alpha_{i j}$ is a vector of individual fixed effects coefficients (or random effects if, e.g., suggested by a Hausmann test), and year $r_{t}$ is the vector of time fixed effects, included to account for variations in the business cycle. The model constant is excluded if all time effects are estimated. In an estimation framework including individual fixed effects, time-invariant variables (like distance) cannot be identified, and are dropped.

Since the spatial interaction model is multiplicative (see Section 2), a typical choice - as for any other multiplicative model, like production functions - is to render it linear in parameters through log-linearization (see, e.g., Lim 1997). In panel applications, the individual fixed effects act as surrogates for the omitted explanatory variables, similarly to the case of international trade models (in which price indices are unobserved; see Anderson and van Wincoop 2003). In this paper, we estimate the spatial interaction model by means of count data regression techniques, that is, in its multiplicative form, in order to account for Jensen's inequality and potential overdispersion. Santos Silva and Tenreyro (2006) have shown, in a widely popular article, that many problems are associated with the log-linearization of multiplicative models, in the presence of heteroskedasticity, and suggested the use of count data regression models. Following Santos Silva and Tenreyro's contribution, Burger et al. (2009) have expanded this discussion by considering a wider family of Poisson-type models. In this regard, the negative binomial model is suggested as a solution to the problem of overdispersion in the data due to unobserved heterogeneity, which hinders the hypothesis at the basis of the Poisson regression model of equal sample mean and variance. Overdispersion phenomena are typical of dyadic data (e.g., in trade, commuting, migration), whose statistical distribution shows a multitude of small flows and a small number of much greater flows. On the basis of the above considerations, we carry out negative binomial two-way fixed effects estimations. Formally, the estimated model can now be written as follows:

$$
T_{i j t}=\exp \left(\alpha_{i j}+y e a r_{t}+X_{i t}+W H S_{i t}+L . W H S_{i t}+X_{j t}+W H S_{j t}+L . W H S_{j t}\right)+\varepsilon_{i j},
$$

where $\varepsilon_{i j}$ is the regression residual for the generic flow from region $i$ to region $j$. A dispersion parameter $\varphi$ is iteratively estimated.

Finally, in order to empirically evaluate the effect of distance, we set up a further model by means of an alternative estimation approach, that is, a panel spatial filtering-based negative binomial model. In this model specification, the individual (pair-level) fixed effects are substituted by two sets of origin and destination dummy variables and a network autocorrelation filter. The former component include, in a common fixed effects manner, all time-invariant information specific to the origin and destination regions (for example, the average level of GDP). The latter component incorporates spatial and network dependence due to omitted variables. Because the fixed effects are moved from the pair-level to the origin- and destination-level, time-invariant bilateral variables can be identified, allowing the estimation of a regression coefficient for the distance variable. ${ }^{5}$ The spatial filter is included in the regression model as a set of eigenvectors related to the chosen spatial weights matrix (see Section 4.1). ${ }^{6}$

The model with distance and spatial filter is the following one:

\footnotetext{
5 Internal distances are computed as $\sqrt{\text { area/ } \pi}$ (see, for example, Leamer 1997; Nitsch 2000).

6 Because the implementation of a panel spatial filtering model is not the main focus of this paper, we refer to Chun and Griffith (2011) and Lionetti and Patuelli (2009) for methodological and implementation details.
} 


$$
T_{i j t}=\exp \left(\alpha_{i}+\alpha_{j}+y e a r_{t}+X_{i t}+W H S_{i t}+L \cdot W H S_{i t}+X_{j t}+W H S_{j t}+L \cdot W H S_{j t}+D_{i j}+\sum_{k} e_{k, i j}\right)+\varepsilon_{i j},
$$

where $\alpha_{i}$ and $\alpha_{j}$ are the origin and destination fixed effects, and $e_{k}$ is the $k$ th network autocorrelation eigenvector selected (and composing the spatial filter).

\subsection{Spatial Sensitivity Analysis: An Interpretative Framework}

In this section, we expand on our second research question, by providing an interpretative framework aimed at understanding how and to what extent the effects of the neighbouring (competing) destinations' WHS endowment on tourism flows (the indirect effect discussed above) may vary depending on the assumptions we make on the tourist's capacity to compare alternative destinations in his/her choice set. In this regard, a spatial sensitivity analysis according to the average number of neighbours $k$ is offered in the paper.

In the case of no neighbours $(k=0)$, all regions are isolated destinations ('islands' in a relational sense). In this case, all additional flows $T$ due to an interest in visiting the new WHS reach the corresponding region independently of the WHS endowment of other regions. In the case of one neighbour $(k=1)$, the regions are not isolated anymore, but have a possible spatial competitor (each), with which they compete on the basis of their WHS endowment. Given that the competitor is perceived by the tourists as 'close', it may now represent a valid alternative, all else being equal. Following the same line of reasoning, in the case of two neighbours $(k=2)$, we hypothesize that the tourists evaluate each destination against its two possible spatial competitors based on WHS, and so on for higher numbers of neighbours.

To build a general model, we make two assumptions. The first assumption is that, although the designation of a WHS could cause a crowding-out effect $(E \geq 0)$ among tourists, as a result of which some of them may dismiss the destination (e.g., the WHS may entail some constraints, costs and limitations in site accessibility), such effect is on average dominated by the positive attractiveness effect $\left(T_{1}>0\right)$ on tourism flows of the designation. Were the direct effect of WHS designation negative $\left(E>T_{1}>0\right)$, applying for it would not be desirable for a rationale destination.

The second assumption concerns the possible spatial interaction effect between neighbouring regions due to their WHS endowment, $T_{2}(k)$, which can be of (i) spatial competition or (ii) spatial complementarity. There is spatial competition when a region's tourism inflows are diminished by the WHS endowment of regions which are perceived as substitute destinations, while there is spatial complementarity when a region receives a benefit, in terms of inflows, from the WHS endowment of 'close' regions (e.g., mutual beneficial effects may derive by trip chaining). Such competition (complementarity) effect may be expected to vary monotonically with the number of neighbours considered, until a threshold is reached after which regions further away are not perceived anymore as substitutes (complements) by tourists.

We can now outline a simple model for the relationship between WHS-induced tourism inflows and the number $k$ of alternative destinations considered by the tourist. We can describe the tourism flows $T$ towards a single destination as depending on other destination characteristics $\left(T_{0}\right.$, given by the generic $X$ in the model) and on WHS endowment, which generates a positive direct effect on inflows $T_{1}>0$. In addition to $T_{1}$, a second effect $T_{2}(k)$ may be included, for the overall interplay of spatial complementarity and competition effects, which depends on the number of neighbours $k$. Let us recall that $T_{2}(0)=0$, while for $k>0, T_{2}(k)>0$ denotes a dominance of spatial complementarity, while $T_{2}(k)<0$ denotes a dominance of spatial competition. Exploiting the Poisson-type estimation framework of the paper, and omitting origin-level variables for notation simplicity, the total tourism inflows of a region $j$ from a region $i$ can be written as:

$$
T_{i j}=\exp \left(T_{0, i j}\right) \exp \left[\left(T_{1}-E\right) W H S_{j}+T_{2}(k) L \cdot W H S_{j}\right]
$$


with $T_{1}>E$, and where $T_{0}$ are inflows due to the control variables, on the scale of the linear predictor. In regression terms, $\beta_{1}=T_{1}-E$ is the regression coefficient estimated for the WHS variable, while $\beta_{2}=T_{2}(k)$ is the coefficient of the spatial lag of $W H S$ (i.e., W $* W H S=L$.WHS). We can now analyse how tourism inflows change conditional to the number of neighbours $k$. If $d T_{1} / d k=$ 0 and $T_{2}(0)=0$, then the sign of the overall effect of WHS on domestic tourism depends only on the assumptions made on the behaviour of $T_{2}(k)$.

We can specify $T_{2}(k)$ as the difference between two separate effects:

$$
T_{2}(k)=T_{2.1}(k)-T_{2.2}(k)
$$

where $T_{2.1}(k) \geq 0$ is spatial complementarity and $T_{2.2}(k) \geq 0$ is spatial competition. By construction, $T_{2.1}(0)=T_{2.2}(0)=0$, and we may hypothesize that both effects increase with $k\left(T_{2.1}{ }^{\prime}(k)>0\right.$ and $\left.T_{2.2}{ }^{\prime}(k)>0\right)$, with $T_{2.1}{ }^{\prime \prime}(k)<0$ and $T_{2.2}{ }^{\prime \prime}(k)>0$, resulting in two functions crossing each other at the value $k^{*}$ that implies an overall null indirect effect $\left[T_{2.1}\left(k^{*}\right)=T_{2.2}\left(k^{*}\right)\right]$, as suggested in Figure $1{ }^{7}$ Here, we hypothesize that competition effects eventually dominate complementarity effects for greater values of $k$.

The above model can explain the mixed empirical evidence in the current literature on the effects of WHS endowment on tourism flows (see Section 3). In fact, even though a region's WHS endowment can potentially attract additional tourists (direct effect; not shown in Figure 1), the indirect effect on tourism flows can be both positive, if it is dominated by spatial complementarity, and negative in the case of a prevailing competitive relationship (spatial competition). Nevertheless, even in the latter case, the overall effect, given $T_{1}$, can be positive, negative or null, depending on the number of spatial competitors (neighbours). Since $T_{2}$ depends on $k, d T_{2} / d k<0$ (for $k$ large enough) implies tendencies towards spatial competition as the number of neighbours considered increases, while tendencies towards spatial complementarity are found in the opposite case. Discussing the most problematic case, that is, the competitive relationship, we note that in general a number of neighbouring destinations $k$ always exists which compensates the direct tourism flows growth induced by WHS increase. Furthermore, if spatial competition is very strong, there will be no advantage whatsoever for destinations deriving from WHS designation, but only potential losses in terms of tourism flows.

The present interpretative framework is particularly fitting for Italian domestic tourism, because of the many WHS, which are well distributed over the different regions (only 2 small regions out of 20 do not have any). Without a precise motivational analysis of the tourists, $T_{1}, E, T_{2.1}$ and $T_{2.2}$ are not directly observable. However, the observed regional tourism flows allow us to test a crucial assumption of the model: if $T_{2}(k)$ is increasing in $k$, which implies tendencies towards a dominance of complementarity, or decreasing in $k$, implying tendencies towards competition.

Furthermore, in the case of spatial competition, we can test: (i) for $k=0$, if the effect of new WHS endowment is positive $\left(T_{1}>E\right)$ o null $\left(T_{1}=E\right)$; and (ii) for $k>0$, if it is possible to identify the number of competitors cancelling out the positive direct effect of WHS endowment, and to justify why with a higher number of competitors the destination can eventually lose tourists. The following section reports our findings.

\footnotetext{
${ }^{7}$ Alternatively, we could hypothesize $T_{2.2}{ }^{\prime \prime}(k)<0$, which would imply one function dominating the other for every value of $k$. We consider the case of the intersecting functions more interesting and we limit ourselves to discussing the latter.
} 


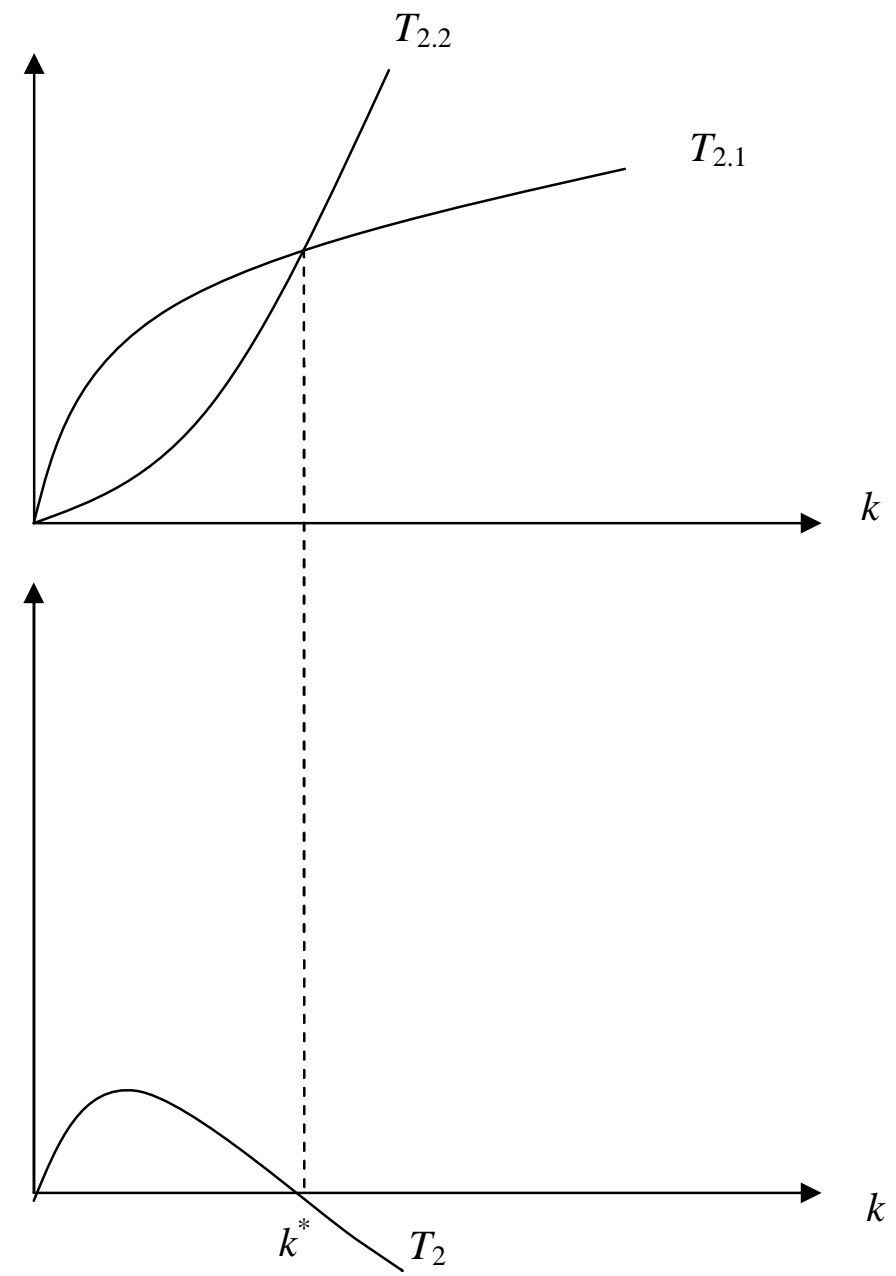

Figure 1. Spatial complementarity and spatial competition effects within $T_{2}(k)$

\section{Empirical Application}

\subsection{Data}

Our empirical application to tourism flows between the 20 Italian regions employs data from the Italian Statistical Agency (ISTAT). The dependent variable, that is, regional arrivals from and to all Italian regions for the period 1998-2009, is provided within the publication 'Statistiche del Turismo', and collected through the accommodation structures survey. Traditional hotel accommodations, as well as complementary accommodations and privately rented houses, are included in the survey.

Our key variable, the number of regional WHS, is obtained directly from UNESCO's World Heritage Convention website (http://whc.unesco.org/), which provides a list of all WHS by country, year of inclusion and nature of the site. All further variables used in this paper are obtained by ISTAT, and are published on: 'Conti Economici Regionali', 'Prezzi al Consumo', and 'Banca Dati Territoriale per le Politiche di Sviluppo'. Table 1 provides a concise description of the variables used in our empirical application. All variables are expressed in logs, aside from WHS and NonBath, which include a share of zeros. 
Table 1. Explanatory variables

\begin{tabular}{|c|c|c|}
\hline Variable & Description & Source \\
\hline WHS & Number of WHS & UNESCO \\
\hline GDP & Regional GDP (1-year lag, in logs) & ISTAT \\
\hline SpecTour & $\begin{array}{l}\text { Specialization in tourism (= share of value added generated from } \\
\text { accommodation and restaurants, commerce, transport, etc.) ( } 2 \text {-year } \\
\text { lag, in logs) }\end{array}$ & ISTAT \\
\hline ExpRecr & $\begin{array}{l}\text { Share of public spending in recreational, cultural and religious } \\
\text { activities ( } 2 \text {-year lag, in logs) }\end{array}$ & ISTAT \\
\hline PricesH\&R & Price index for hotels and restaurants (in logs) & ISTAT \\
\hline Pop & Regional population (in logs) & ISTAT \\
\hline CrimDiff & $\begin{array}{l}\text { Small crime index (= thefts and robberies x 1,000 inhabitants) (1-year } \\
\text { lag, in logs) }\end{array}$ & ISTAT \\
\hline CrimVio & $\begin{array}{l}\text { Violent crime index (= violent crimes x 10,000 inhabitants) (2-year } \\
\text { lag, in logs) }\end{array}$ & ISTAT \\
\hline SatisTrain & $\begin{array}{l}\text { Train service satisfaction index (= share of customers who declare to } \\
\text { be satisfied with train service) (in logs) }\end{array}$ & ISTAT \\
\hline CultDem & $\begin{array}{l}\text { Cultural demand index (= visitors to state antiquities and arts } \\
\text { museums x institute) (1-year lag, in logs) }\end{array}$ & ISTAT \\
\hline DiffShows & $\begin{array}{l}\text { Diffusion of theatrical and musical shows (= theatrical and musical } \\
\text { shows tickets sold x } 100 \text { inhabitants) (1-year lag, in logs) }\end{array}$ & ISTAT \\
\hline NonBath & $\begin{array}{l}\text { Non bathable coast (= share of coast kms which are non-bathable due } \\
\text { to pollution) (1-year lag) }\end{array}$ & ISTAT \\
\hline OffSeas & $\begin{array}{l}\text { Deseasoning index (= overnight stays in off-season months } \mathrm{x} \\
\text { inhabitant) }(1 \text {-year lag, in logs })\end{array}$ & ISTAT \\
\hline Distance & Distance between regional centroids (in km, in logs) & $\begin{array}{l}\text { Own } \\
\text { calculation }\end{array}$ \\
\hline
\end{tabular}

\subsection{Model Results}

We start by estimating a benchmark model, that is, a standard tourism spatial interaction model, including only the control variables described in Sections 4.1 and 5.1. Empirical estimates are provided in Table 2.

Model (1) in Table 2 confirms most of our basic assumptions. Regional inflows are positively influenced by supply factors, like the quality of the museum offer (CultDem) and the diffusion of cultural events (DiffShows), and negatively influenced by the level of prices of the restoration/accommodation sector (PricesH\&R). Furthermore, regions which deseasonalize (OffSeas) enjoy greater inflows. Public spending in recreational activities (ExpRecr), instead, does not appear to have a significant impact. Finally, greater inflows are associated with lower GDP, suggesting that tourists look for less developed, more relaxing destinations. With regard to the regional outflows, GDP, which conditional to population identifies per capita income, is surprisingly not significant as a push effect. Crime levels appear to have a role also in pushing tourists (out), since they positively affect outflows (CrimVio and CrimDiff), implying that residents of at-risk areas tend to get away in search of safer destinations. The residents of regions specialized in tourism (SpecTour) appear to have a higher propensity to travel, possibly according to an 'addiction to tourism' effect or as a refuge from the summer crowding. 
Table 2. Empirical estimates

\begin{tabular}{|c|c|c|c|c|c|c|}
\hline & $\begin{array}{l}\text { Estimate } \\
\text { (Std error) } \\
\text { (1) }\end{array}$ & p-value & $\begin{array}{l}\text { Estimate } \\
\text { (Std error) } \\
\text { (2) }\end{array}$ & p-value & $\begin{array}{l}\text { Estimate } \\
\text { (Std error) } \\
\text { (3) }\end{array}$ & p-value \\
\hline GDP orig & $0.2101(0.3498)$ & 0.5481 & $0.1834(0.3481)$ & 0.5983 & $0.3470(0.6337)$ & 0.5840 \\
\hline GDP dest & $-2.2471(0.3636)$ & $<0.0001$ & $-2.4442(0.3670)$ & $<0.0001$ & $-2.2774(0.6524)$ & 0.0005 \\
\hline SpecTour orig & $0.2823(0.1098)$ & 0.0101 & $0.2973(0.3670)$ & 0.0070 & $0.3177(0.2408)$ & 0.1870 \\
\hline SpecTour dest & $0.3686(0.1373)$ & 0.0072 & $0.1753(0.1407)$ & 0.2128 & $0.1701(0.2692)$ & 0.5275 \\
\hline ExpRecr orig & $0.0846(0.0670)$ & 0.2068 & $0.0422(0.0667)$ & 0.5272 & $0.0469(0.1254)$ & 0.7085 \\
\hline ExpRecr dest & $-0.0681(0.0552)$ & 0.2174 & $-0.0832(0.0595)$ & 0.1617 & $-0.0632(0.1246)$ & 0.6118 \\
\hline PricesH\&R orig & $0.2101(0.2610)$ & 0.4207 & $0.2307(0.2646)$ & 0.3832 & $0.3454(0.4700)$ & 0.4624 \\
\hline PricesH\&R dest & $-0.8296(0.2405)$ & 0.0006 & $-1.1275(0.2453)$ & $<0.0001$ & $-1.3658(0.4594)$ & 0.0030 \\
\hline Pop orig & $-0.4803(0.4571)$ & 0.2933 & $-0.1232(0.4522)$ & 0.7853 & $-0.7002(0.7332)$ & 0.3396 \\
\hline Pop dest & $0.3004(0.2860)$ & 0.2936 & $0.6503(0.2590)$ & 0.0120 & $0.3365(0.5801)$ & 0.5619 \\
\hline CrimDiff orig & $0.1159(0.0533)$ & 0.0298 & $0.1153(0.0533)$ & 0.0304 & $0.1139(0.0992)$ & 0.2508 \\
\hline CrimDiff dest & $-0.0237(0.0279)$ & 0.3966 & $-0.0044(0.0280)$ & 0.8755 & $0.0117(0.0733)$ & 0.8731 \\
\hline CrimVio orig & $0.0522(0.0264)$ & 0.0480 & $0.0563(0.0270)$ & 0.0373 & $0.0577(0.0492)$ & 0.2412 \\
\hline CrimVio dest & $-0.0214(0.0251)$ & 0.3951 & $0.0099(0.0255)$ & 0.6987 & $-0.0010(0.0500)$ & 0.9837 \\
\hline SatisTrain orig & $0.0696(0.0450)$ & 0.1226 & $0.0292(0.0460)$ & 0.5251 & $0.0677(0.1073)$ & 0.5282 \\
\hline SatisTrain dest & $0.0551(0.0514)$ & 0.2837 & $0.0627(0.0499)$ & 0.2088 & $0.1059(0.1059)$ & 0.3176 \\
\hline CultDem orig & $-0.0356(0.0222)$ & 0.1089 & $-0.0305(0.0223)$ & 0.1722 & $-0.0234(0.0456)$ & 0.6080 \\
\hline CultDem dest & $0.1879(0.0223)$ & $<0.0001$ & $0.2073(0.0233)$ & $<0.0001$ & $0.1971(0.0452)$ & $<0.0001$ \\
\hline DiffShows orig & $0.0574(0.0390)$ & 0.1409 & $0.0656(0.0391)$ & 0.0929 & $0.0818(0.0689)$ & 0.2350 \\
\hline DiffShows dest & $0.0967(0.0309)$ & 0.0017 & $0.0868(0.0317)$ & 0.0062 & $0.0818(0.0624)$ & 0.1902 \\
\hline NonBath orig & $0.0007(0.0027)$ & 0.7851 & $0.0011(0.0027)$ & 0.6691 & $0.0003(0.0048)$ & 0.9547 \\
\hline NonBath dest & $0.0006(0.0027)$ & 0.8140 & $0.0016(0.0028)$ & 0.5562 & $0.0016(0.0060)$ & 0.7864 \\
\hline OffSeas orig & $-0.0033(0.0393)$ & 0.9335 & $0.0020(0.0389)$ & 0.9599 & $0.0230(0.0778)$ & 0.7677 \\
\hline OffSeas dest & $0.4093(0.0521)$ & $<0.0001$ & $0.3915(0.0514)$ & $<0.0001$ & $0.3541(0.0927)$ & 0.0001 \\
\hline WHS orig & - & - & $-0.0164(0.0079)$ & 0.0387 & $-0.0203(0.0159)$ & 0.2009 \\
\hline L.WHS orig & - & - & $-0.0451(0.0198)$ & 0.0227 & $-0.0516(0.0364)$ & 0.1567 \\
\hline WHS dest & - & - & $0.0355(0.0067)$ & $<0.0001$ & $0.0420(0.0147)$ & 0.0044 \\
\hline L.WHS dest & - & - & $-0.1035(0.0204)$ & $<0.0001$ & $-0.0983(0.0365)$ & 0.0071 \\
\hline Distance & - & - & - & - & $-1.0165(0.0362)$ & $<0.0001$ \\
\hline AIC & 71705 & - & 71660 & - & 75689 & - \\
\hline BIC & 74136 & - & 74116 & - & 76369 & - \\
\hline Res. dof & 2977 & - & 2973 & - & 3263 & - \\
\hline $\begin{array}{l}\text { McFadden's } \\
\text { pseudo- } R^{2}\end{array}$ & 0.4068 & - & 0.4073 & & 0.1199 & - \\
\hline $\begin{array}{l}\text { ANOVA } \\
\left(\chi^{2} \text { LR test }\right)\end{array}$ & - & - & 52.9132 & $<0.0001$ & - & - \\
\hline
\end{tabular}

We can now augment the benchmark model by including our variables of interest, that is, WHS and L.WHS, again evaluated both at the origins and at the destinations. Model (2) in Table 2 provides empirical estimates for Equation (5). The inclusion of the WHS variables allows to retain the findings of Model (1), while providing evidence for an effect of WHS endowment on tourism flows. We find that, with regard to the destinations, WHS are positively associated with inflows (this being the direct effect of WHS on tourism, and including, if existent, crowding-out effects). An increase of one WHS, for a generic destination, would imply, with a 95 per cent confidence interval, an inflows increase between 2.9 and 4.3 per cent $[\exp (0.0355)=1.0361]$. The effects of the WHS endowment of neighbouring destinations (the indirect effects of complementarity and competition) are also found to be significant, but negative, suggesting the existence of spatial competition between contiguous regions induced by WHS endowment. Clearly, a complementarity effect could exist as well, but appears to be dominated by the competition effect. Our finding implies that an increase of one WHS, on average, in the surrounding regions of a generic destination 
(an acceptable assumption in the long run) would lead to a decrease of arrivals, for the above destination, of about 10 per cent $[\exp (-0.1035)=-0.9017]$. As the marginal effects of 'WHS dest' and 'L.WHS dest' cannot be considered separately, we conclude that, for an increase of one WHS in every region, the overall effect on tourism flows would be negative (around 6 per cent) when competing destinations are defined by shared borders.

With regard to the origin regions, we find a negative and significant sign (suggesting that outflows would decrease with the increase in WHS endowment) for both the direct and the indirect effects, reinforcing the hypothesis that the availability of WHS near the tourist's residence may lead to substitution between hotel arrivals (traditional tourism) and daily excursions, both within the residence region and to nearby (alternative) destinations. The decrease of outflows is numerically consistent with the decrease of inflows discussed above. From a statistical perspective, Model (2) improves significantly on the reliability of the benchmark model [Model (1)], as suggested by the improvements in terms of AIC, BIC and pseudo- $R^{2}$, as well as by a $\chi^{2}$-based likelihood ratio (LR) test between the two models, which is highly significant.

Finally, Model (3) presents the results from an eigenvector spatial filtering model specification [Equation (6)], carried out in order to evaluate the tourists' demand elasticity with respect to distance. With regard to the control variables, only selected destination-level variables are found to be significant (GDP, PricesH\&R, CultDem, and OffSeas). The WHS and L.WHS variables, again for destination regions only, are significant and similar in estimated effect size to Model (2). The regression coefficient for the effect of distance is close to 1 (a unitary elasticity), suggesting, for example, that all else being equal, a destination region being 20 per cent more far away than another from a specified origin region will receive 20 per cent less tourists. ${ }^{8}$

\subsection{Spatial Sensitivity Analysis}

The analyses presented in the preceding section are based on a generic assumption of spatial competition/complementarity happening along regional borders. In particular, it is assumed that in evaluating the attractiveness of each region the tourist considers all other regions with which the destination shares a border (rook contiguity definition of proximity). This assumption is a common practice in the spatial econometric literature, but can be explicitly tested against alternative specifications of the spatial weights matrix.

We carry out a spatial sensitivity analysis to test the robustness of our findings to different hypotheses on the nature and geographical extent of spatial interaction, according to the interpretative framework described in Section 4.3. In particular, we aim to test to what extent the overall effect of WHS endowment on tourism flows may vary, in particular with regard to the (indirect) effect of WHS endowment in neighbouring regions and the average number of possible spatial competitors.

From a methodological viewpoint, the effect size and statistical significance of the indirect effect of WHS endowment may be sensitive to the definition of 'neighbours' used, that is, to the choice of the spatial weights matrix W. In Models (2) and (3) of Table 2, we define $\mathbf{W}$ by rook contiguity. According to this criterion, the average number of neighbours per region is 3.1, ranging from 0 to 6 .

To carry out a spatial sensitivity analysis, we test different specifications of $\mathbf{W}$ for an increasing number of assigned neighbours, according to two additional definitions of proximity:

- $k$-nearest neighbours, based on Great Circle distance, for $k=0,1, \ldots, 4$;

- distance thresholds, based on distance bands computed as $h^{*} \min ($ dist $)$, with $h=0,2, \ldots, 4$.

8 A sensitivity analysis testing polynomial specifications for the distance term shows that a cubic specification provides slight fitting advantages (for example, in terms of BIC). The negative-positive-negative signs for the three terms of the polynomial suggest that a destination's distance from the tourist's residence region becomes a positive tourism reinforcing factor only after a certain threshold (after which the destination appears to be 'exotic'), and up to a second threshold level, after which the distance deterrence effect again becomes dominant. 
Table 3 provides the empirical estimates for the effect of the destinations' WHS endowment on tourist inflows according to the $k$-nearest neighbours criterion, and presents the direct and indirect effect estimates (for variables 'WHS dest' and 'L.WHS dest'). In addition to effect size estimates, Table 3 provides AIC values and two $\chi^{2}$-based likelihood ratio (LR) tests against (i) the hypothesis of equivalence between the $k>0$ models and the $k=0$ model (i.e., with no L.WHS variables), and (ii) the hypothesis that the overall effect of WHS endowment is null.

Table 3. $k$-nearest neighbours results for WHS dest and L.WHS dest

\begin{tabular}{llllll}
\hline$k$-nearest neighbours & 0 & 1 & 2 & 3 & 4 \\
\hline WHS dest & $0.0395 * * *$ & $0.0394 * * *$ & $0.0390^{* * *}$ & $0.0372 * * *$ & $0.0357 * * *$ \\
L.WHS dest & - & -0.0153 & -0.0210 & -0.0320 & $-0.0731^{* * *}$ \\
AIC & 71762 & 71758 & 71757 & 71755 & 71736 \\
LR $\left(\chi^{2}\right)$ test & - & $8.04 * *$ & $9.14^{* *}$ & $10.62^{* * *}$ & $30.41^{* * *}$ \\
$H_{0}: k=0$ & - & & & 1.48 \\
LR $\left(\chi^{2}\right)$ test & - & - & - & - & \\
$H_{0}: \beta_{\text {WHS dest }}+\beta_{\text {L.WHS dest }}=0$ & & & & & \\
\hline
\end{tabular}

Table 3 shows that, when applying a $k$-nearest neighbours definition of proximity, the direct effect of WHS endowment is positive and numerically stable over estimations (around 4 per cent), confirming the results of Section 5.2. The WHS indirect effect is negative and increases in size with the number of neighbours, although it becomes significant only with $k \geq 4$. The LR $\chi^{2}$ tests against the $k=0$ model always reject the hypothesis of equivalence, suggesting that the characteristics of competing destinations should indeed be considered in our modelling framework. Moreover, the LR test against the hypothesis that the overall effect of WHS endowment is null, which is not computed when a direct effect only is found, is not significant for $k=4$, that is, when 4 neighbours per region are considered, the direct effect of WHS endowment is cancelled by the (negative) effect of WHSbased competition.

Finally, Table 4 provides the estimated effect sizes of WHS endowment according to distancethreshold models, with $\min ($ distance) $=67 \mathrm{~km}$. Several distance bands (for $h=0,2,3,4$ ) are tested, corresponding each to a different neighbours list.

Table 4. Distance-threshold results for WHS dest and L.WHS dest

\begin{tabular}{llccc}
\hline Distance threshold & 0 & $\begin{array}{l}2 * \min (\text { dist }) \\
(1.3 \text { neigh. }\end{array}$ & $\begin{array}{l}3 * \min (\text { dist }) \\
(3.3 \text { neigh. }\end{array}$ & $\begin{array}{c}4 * \text { min(dist) } \\
(5.1 \text { neigh. }\end{array}$ \\
\hline WHS dest & $0.0395^{* * *}$ & $0.0366^{* * *}$ & $0.0371^{* * *}$ & $0.0398^{* * *}$ \\
L.WHS dest & - & $-0.0317^{* *}$ & $-0.0484^{* *}$ & $-0.1386^{* * *}$ \\
AIC & 71762 & 71757 & 71749 & 71714 \\
LR $\left(\chi^{2}\right)$ test & - & $8.83^{* *}$ & $16.80^{* * *}$ & $52.51^{* * *}$ \\
$H_{0}: k=0$ & & & & \\
LR $\left(\chi^{2}\right)$ test & - & 0.07 & 0.20 & $13.99 * * *$ \\
$H_{0}: \beta_{\text {WHS dest }}+\beta_{\text {L.WHS dest }}=0$ & & & & \\
\hline
\end{tabular}

The distance-threshold models show again a positive and stable direct effect of WHS endowment (and always close to 4 per cent), while the indirect effect is significant and negative starting from the first bandwidth, and increasing with distance, like in the $k$-nearest neighbours case. The LR tests against the neighbourless model always reject the hypothesis of equivalence, as before, while the LR test against the hypothesis of null overall effect of WHS endowment is not significant for the 
first two bandwidths tested, while it becomes significant for $h \geq 4$, for an overall negative effect over greater distances.

Finally, with regard to the interpretative model provided in Section 4.3 , we can note that the hypothesis of a tendency towards the dominance of spatial competition over spatial complementarity is confirmed. However, our results, showing first a non-significant indirect effect and then a negative one, do not allow us to shed light on the possibility, suggested in Figure 1, of a sign inversion of the indirect effect over increasing values of $k$, and therefore on the sign of $T_{2.2}{ }^{\prime \prime}(k)<0$.

In summary, our sensitivity analysis shows that the assumption made over the geographical extent at which regions may compete for tourists in terms of their WHS endowment matters. Among all the models tested above, the model with the best fit (in terms of AIC) is the one with the largest distance bandwidth, although Model (2) from Table 2, based on rook contiguity, has the best fit overall. Our findings suggest that, when comparing destinations on the basis of their WHS endowment, the tourist uses a potentially large number of alternative destinations.

\section{Conclusions}

In this paper, we analysed the relationship between domestic tourism and cultural endowment measured in terms of the number of sites enlisted in UNESCO's World Heritage List (WHS). We carried out an empirical application for 20 Italian regions for the years 1998-2009, employing data on the interregional tourism flows recorded as arrivals in hotels and other types of accommodation structures.

Our objectives can be framed within the ongoing debate on the relevance of cultural - and more specifically WHS - endowment for tourism. Our contribution appears to support the view that such a relationship exists, and that its numerical extent is non-negligible.

The first research question we aimed to answer was whether the regional WHS endowment affects tourism flows. On the one hand, we find that regions which are endowed in WHS are able to attract a greater number of tourists, all else being equal. More in detail, an increase of one WHS in a region's endowment implies a 4 per cent increase in tourist inflows. On the other hand, we found a negative effect of WHS endowment on regional outflows, that is, on the emissiveness of the tourists' residence regions. The estimated effect, around -1.6 per cent is most likely due to substitution between hotel arrivals (traditional - and recordable - tourism) and daily excursions.

Our second research question regarded the spatial behaviour of tourists, and the potential substitution or complementarity between tourism destinations. We found that the WHS endowment of the regions surrounding a hypothetical destination (i.e., sharing a border with it) has a negative effect on its inflows of tourists. We estimated this effect at about 10 per cent for an average increase of one WHS in a destination's neighbours. We may then speculate that, consistently with a competing destinations framework (Fotheringham 1983), tourists appear to consider, in forming their travelling choices, the WHS endowment of alternative destinations, generating a phenomenon of spatial substitution. The overall effect of a generalized increase of one unit in the WHS endowment of the regions would then lead - on average - to a negative balance ( -6 per cent) in inflows. Consistently with the above results, we find that tourism outflows appear to be constrained also by the endowment of the origin region's neighbours.

Finally, in order to investigate the robustness of our findings to different hypotheses on the nature and geographical extent of spatial interaction, we carried out a spatial sensitivity analysis. In particular, we applied two further definitions of proximity, according to the $k$-nearest neighbours method and to distance thresholds.

With regard to the effect of WHS endowment on inflows (i.e., towards the destination regions), the result of a positive direct effect was confirmed in all cases. The negative indirect effect of the WHS endowment of neighbouring destinations (up to -13 per cent) was confirmed as well, showing in particular that the spatial competition effect becomes significant when a greater geographical 
extent is considered. The overall effect is therefore: first (i) positive and equal to about 4 per cent (when only 1 or 2 neighbouring regions are considered), then (ii) non-significantly different from zero (when the number of spatial competitors is 3), and finally (iii) negative ( -9 per cent for the greatest distance bandwidth considered). This result may help explaining the mixed empirical evidence found in the literature, and suggests that spatial competition appears to dominate spatial complementarity (i.e., a cultural district effect is not observable, at least at the regional spatial scale).

Altogether, we can conclude that the spatial sensitivity analysis confirmed the robustness of our results. Furthermore, for all the definitions of proximity tested, the models including indirect effects outperformed in terms of AIC the model with only direct effects, confirming the empirical relevance of our second research question.

From a policy viewpoint, our findings have two main implications: (1) WHS endowment does appear to influence arrivals to tourism destinations for Italian domestic tourism, ${ }^{9}$ providing a justification for local policymakers' lobbying towards the national government for obtaining UNESCO designations; (2) however, spatial competition may reduce the positive direct effect down to an overall negative effect, once more alternatives are considered and more sites are assigned to competing destinations as well, suggesting that the desirability of WHS designation depends on the expected spatial extent of competition. This last result strengthens the importance of WHS endowment, since it implies that competition among regions on the basis of WHS can be justified. In fact, given that the positive effects of trip-chaining are outweighed by spatial competition, regions could indeed use WHS designations to gain competitive advantages over other regions, which outlines the critical role of regional tourism promotion agencies.

Further improvements, from a methodological viewpoint, could involve the estimation of our model in a dynamic panel framework, to account for inertia mechanisms, as well as in its doublyconstrained form, so as to provide a view on the spatial substitution/complementarity effects under the hypothesis of constant overall tourism flows. From an empirical viewpoint, it would be desirable to augment the model specification by introducing physical variables (e.g., kms of coastline, mean elevation) and further proxies of cultural offer, in order to further improve the identification of the WHS endowment's contribution to tourism.

\section{Acknowledgments}

The authors wish to thank for useful comments session participants and seminar attendees at the 2011 NECTAR Conference (Antwerp), the 51st European Congress of the Regional Science Association International (Barcelona), the Fifth European Workshop on Applied Cultural Economics (Dublin), the SNSF International Exploratory Workshop on 'Advances in the Statistical Modelling of Spatial Interaction Data' (Lugano), the Toulouse School of Economics of the Université Toulouse 1 Capitole (Toulouse), the Workshop on 'The Economics and Management of Leisure, Travel and Tourism' (Rimini), and the Workshop on 'UNESCO World Heritage: Economic and Policy Issues' (Turin).

\section{References}

van der Aa, B. J. M. (2005). Preserving the Heritage of Humanity? Obtaining World Heritage Status and the Impacts of Listing. Unpublished Ph.D. thesis, University of Groningen, Groningen.

Alzua, A., O'Leary, J. T. and Morrison, A. (1998). Cultural and Heritage Tourism: Identifying

Niches for International Travellers. Journal of tourism studies, 9(2), 2-13.

9 Our results might not carry over to international tourism. 
Anderson, J. E. (1979). A Theoretical Foundation for the Gravity Equation. The American Economic Review, 69(1), 106-16.

Anderson, J. E. and van Wincoop, E. (2003). Gravity with Gravitas: A Solution to the Border Puzzle. American Economic Review, 93(1), 170-92.

Armstrong, G. W. G. (1972). International Tourism: Coming or Going: The Methodological Problems of Forecasting. Futures, 4(2), 115-25.

Ashworth, G. J. and Tunbridge, J. E. (1990). The Tourist-Historic City. London New York: Belhaven Press.

Baier, S. L. and Bergstrand, J. H. (2009). Bonus Vetus OLS: A Simple Method for Approximating International Trade-Cost Effects Using the Gravity Equation. Journal of International Economics, 77(1), 77-85.

Barry, K. and O'Hagan, J. (1972). An Econometric Study of British Tourist Expenditure in Ireland. The Economic and Social Review, 5, 143-61.

Becker, G. S. (1996). Accounting for Tastes. Cambridge: Harvard University Press.

Blacik, L. (2007). A Critical Assessment of the Impact of World Heritage Site Designation in SubSaharan Africa (ISP Collection. Paper No. 150).

Bond, D., Cohen, B. and Schachter, G. (1977). The Spatial Distribution of Tourism Demand and Exchange Rate Variation: OECD European Countries. Tourism Review, 32(1), 13-17.

Bové Sans, M. and Laguado Ramírez, R. (2011). Image Destination Analysis for Tarragona's Cultural Heritage. Paper presented at the The Economics and Management of Leisure, Travel and Tourism, Rimini.

Buckley, R. (2004). The Effects of World Heritage Listing on Tourism to Australian National Parks. Journal of Sustainable Tourism, 12(1), 70-84.

Burger, M., van Oort, F. and Linders, G.-J. (2009). On the Specification of the Gravity Model of Trade: Zeros, Excess Zeros and Zero-inflated Estimation. Spatial Economic Analysis, 4(2), 16790.

Calantone, R. J., Di Benedetto, C. A. and Bojanic, D. (1987). A Comprehensive Review Of The Tourism Forecasting Literature. Journal of Travel Research, 26(2), 28-39.

Candela, G. and Figini, P. (2012). The Economics of Tourism Destinations. Berlin London: Springer.

Carr, E. A. J. (1994). Tourism and Heritage: The Pressures and Challenges of the 1990s. In G. J. Ashworth and P. J. Larkham (Eds.), Building a New Heritage: Tourism, Culture, and Identity in the New Europe (pp. 69-89). London New York: Routledge.

Cellini, R. (2011). Is UNESCO recognition effective in fostering tourism? A comment on Yang, Lin and Han. Tourism Management, 32(2), 452-54.

Cellini, R. and Cuccia, T. (2009). Museum and Monument Attendance and Tourism Fow: A Time Series Analysis Approach (MPRA Paper No. 18908). Munich: Munich Personal RePEc Archive.

Chun, Y. and Griffith, D. A. (2011). Modeling Network Autocorrelation in Space-Time Migration Flow Data: An Eigenvector Spatial Filtering Approach. Annals of the Association of American Geographers, 101(3), 523-36.

Colwell, P. F. (1982). Central Place Theory and the Simple Economic Foundations of the Gravity Model. Journal of Regional Science, 22(4), 541-46.

Crampon, L. J. and Tan, K. T. (1973). A Model of Tourism Flow into the Pacific. Tourism Review, 28(3), 98-104.

Cuccia, T. and Cellini, R. (2007). Is Cultural Heritage Really Important for Tourists? A Contingent Rating Study. Applied Economics, 39(2), 261-71.

Dean, A., Morgan, D. and Tan, T. E. (2002). Service Quality and Customers' Willingness to Pay More for Travel Services. Journal of Travel \& Tourism Marketing, 12(2-3), 95-110.

Dhariwal, R. (2005). Tourist Arrivals in India: How Important Are Domestic Disorders? Tourism Economics, 11(2), 185-205.

Di Giovine, M. A. (2009). The Heritage-scape: UNESCO, World Heritage, and Tourism. Lanham: Lexington Books. 
Di Lascio, F. M. L., Giannerini, S., Scorcu, A. E. and Candela, G. (2011). Cultural Tourism and Temporary Art Exhibitions in Italy: A Panel Data Analysis (Mimeo). Bologna: Universsity of Bologna.

Dritsakis, N. (2004). Cointegration Analysis of German and British Tourism Demand for Greece. Tourism Management, 25(1), 111-19.

Drost, A. (1996). Developing Sustainable Tourism for World Heritage Sites. Annals of Tourism Research, 23(2), 479-84.

Edwards, A. D. (1976). International Tourism Development: Forecasts to 1985. London: Economist Intelligence Unit.

Eilat, Y. and Einav, L. (2004). Determinants of International Tourism: A Three-Dimensional Panel Data Analysis. Applied Economics, 36(12), 1315-27.

Fotheringham, A. S. (1983). A New Set of Spatial-Interaction Models: The Theory of Competing Destinations. Environment and Planning A, 15, 15-36.

Frey, B. S. and Steiner, L. (2011). World Heritage List: Does it Make Sense? International Journal of Cultural Policy, 17(5), 555-73.

Fujii, E. T., Khaled, M. and Mak, J. (1985). An Almost Ideal Demand System for Visitor Expenditures. Journal of Transport Economics and Policy, 19(2), 161-71.

Fujii, E. T., Khaled, M. and Mak, J. (1987). An Empirical Comparison of Systems of Demand Equations for Tourist Expenditures in Resort Destinations. Philippine Review of Economics, 24(1-2), 79-102.

Gamboni, D. (2001). World Heritage: Shield or Target? Conservation, 16(2), 5-11.

Gardini, A. (1998). L’Analisi della Domanda e della Produzione di Servizi Turistici. Bologna: CLUEB.

Gerakis, A. S. (1965). Effects of Exchange-Rate Devaluations and Revaluations on Receipts from Tourism. Staff Papers - International Monetary Fund, 12(3), 365-84.

Hall, C. M. (2006). Implementing the World Heritage Convention: What Happens after Listing? In A. Leask and A. Fyall (Eds.), Managing World Heritage Sites (pp. 20-34). Oxford Burlington: Butterworth-Heinemann.

Hall, C. M. and Piggin, R. (2001). Tourism and World Heritage in OECD countries. Tourism Recreation Research, 26(1), 103-05.

Haynes, K. E. and Fotheringham, A. S. (1984). Gravity and Spatial Interaction Models. Beverly Hills: Sage Publications.

Keum, K. (2010). Tourism Flows and Trade Theory: A Panel Data Analysis with the Gravity Model. The Annals of Regional Science, 44(3), 541-57.

Khadaroo, J. and Seetanah, B. (2008). The Role of Transport Infrastructure in International Tourism Development: A Gravity Model Approach. Tourism Management, 29(5), 831-40.

Kim, S.-I. and Fesenmaier, D. R. (1990). Evaluating Spatial Structure Effects in Recreation Travel. Leisure Sciences, 12(4), 367-81.

de la Mata, T. and Llano-Verduras, C. (2011). Spatial Pattern and Domestic Tourism: An Econometric Analysis Using inter-Regional Monetary Flows by Type of Journey*. Papers in Regional Science, (forthcoming).

Leamer, E. E. (1997). Access to Western Markets and Eastern Effort. In S. Zecchini (Ed.), Lessons from the Economic Transition: Central and Eastern Europe in the 1990s (pp. 503-26). Norwell Dordrecht: Kluwer Academic Publishers.

Leamer, E. E. and Levinsohn, J. (1995). International Trade Theory: The Evidence. In G. M. Grossman and K. Rogoff (Eds.), Handbook of International Economics (Vol. III, pp. 1339-94). Amsterdam Oxford: North Holland.

Li, M., Wu, B. and Cai, L. (2008). Tourism Development of World Heritage Sites in China: A Geographic Perspective. Tourism Management, 29(2), 308-19.

Light, D. (2000). Gazing on Communism: Heritage Tourism and post-Communist Identities in Germany, Hungary and Romania. Tourism Geographies, 2(2), 157-76. 
Lim, C. (1997). Review of International Tourism Demand Models. Annals of Tourism Research, 24(4), 835-49.

Linnemann, H. (1966). An Econometric Study of International Trade Flows. Amsterdam: NorthHolland.

Lionetti, S. and Patuelli, R. (2009). Trading Cultural Goods in the Era of Digital Piracy (Quaderno della Facolta' di Scienze Economiche No. 09-07). Lugano: University of Lugano.

Lowry, I. S. (1966). Migration and Metropolitan Growth: Two Analytical Models. San Francisco, CA: Chandler.

Malamud, B. (1973). Gravity Model Calibration of Tourist Travel to Las Vegas. Journal of Leisure Research, 5(1), 13-33.

Massidda, C. and Etzo, I. (2011). The Determinants of Italian Domestic Tourism: A Panel Data Analysis. Tourism Management, (forthcoming).

McAllister, D. M. and Klett, F. R. (1976). A Modified Gravity Model of Regional Recreation Activity with an Application to Ski Trips. Journal of Leisure Research, 8(1), 21-34.

McKercher, B. and du Cros, H. (2002). Cultural Tourism: The Partnership between Tourism and Cultural Heritage Management. New York: Haworth Hospitality Press.

Meskell, L. (2002). Negative Heritage and Past Mastering in Archaeology. Anthropological Quarterly, 75(3), 557-74.

Mossetto, G. (1994). The Economic Dilemma of Heritage Preservation. In A. Peacock and I. Rizzo (Eds.), Cultural Economics and Cultural Policies (pp. 81-96). Dordrecht Boston: Kluwer Academic Publishers.

Nicolau, J. L. (2008). Characterizing Tourist Sensitivity to Distance. Journal of Travel Research, 47(1), 43-52.

Nitsch, V. (2000). National Borders and International Trade: Evidence from the European Union. Canadian Journal of Economics/Revue canadienne d'économique, 33(4), 1091-105.

Pierret, F. (2011). Some points on Domestic Tourism. from http://unwto.org/en/opinion/somepoints-domestic-tourism

Pocock, D. (1997). Some Reflections on World Heritage. Area, 29(3), 260-68.

Pöyhönen, P. (1963). A Tentative Model for the Volume of Trade between Countries. Weltwirtschaftliches Archiv, 90, 93-100.

PriceWaterhouseCoopers LLP. (2007). The Costs and Benefits of World Heritage Site Status in the UK: Case Studies. London: Department for Culture, Media and Sport.

Research Consulting Ltd and Trends Business Research Ltd. (2009). World Heritage Status: Is there Opportunity for Economic Gain? Kendal: Lake District World Heritage Project.

Santos Silva, J. M. C. and Tenreyro, S. (2006). The Log of Gravity. Review of Economics and Statistics, 88(4), 641-58.

Saunders, P. R., Senter, H. F. and Jarvis, J. P. (1981). Forecasting Recreation Demand in the Upper Savannah River Basin. Annals of Tourism Research, 8(2), 236-56.

Sen, A. and Smith, T. E. (1995). Gravity Models of Spatial Interaction Behavior. Heidelberg and New York: Springer.

Shackley, M. L. (1998). Visitor Management: Case Studies from World Heritage Sites. Oxford Boston: Butterworth Heinemann.

Sheldon, P. J. and Var, T. (1985). Tourism Forecasting: A Review of Empirical Research. Journal of Forecasting, 4(2), 183-95.

Soares, J. O., Neves, J. and Fernandes, F. (2007). The Impact of World Heritage Classification on the Development of Tourist Destinations: The Sintra Case Study. Paper presented at the Advances in Tourism Economics, Vila Nova de Sto André.

Swart, W. W., Var, T. and Gearing, C. E. (1978). Operations Research Applications to Tourism. Annals of Tourism Research, 5(4), 414-28.

Thorsell, J. and Sigaty, T. (2001). Visitor Human Use in World Heritage Natural Sites: A Global Inventory. Tourism Recreation Research, 26(1), 85-101. 
Tinbergen, J. (1962). Shaping the World Economy: Suggestions for an International Economic Policy. New York: Twentieth Century Fund.

UNESCO. (2011). World Heritage List. from http://whc.unesco.org/en/list

UNESCO. (2012). The World Heritage Convention. Retrieved 19 April, 2012, from http://whc.unesco.org/en/convention\#Benefits-of-Ratification

UNWTO. (1994). Recommendations on Tourism Statistics. New York: UNWTO.

UNWTO. (2011). Facts and Figures: Information, Analysis and Know-How. from http://www.unwto.org/facts/menu.html

Uysal, M. and Crompton, J. L. (1985). An Overview of Approaches Used to Forecast Tourism Demand. Journal of Travel Research, 23(4), 7-15.

VanBlarcom, B. L. and Kayahan, C. (2011). Assessing the Economic Impact of a UNESCO World Heritage Designation. Journal of Heritage Tourism, 6(2), 143-64.

Vietze, C. (2008). Cultural Effects on Inbound Tourism into the USA: A Gravity Approach (Jena Economic Research Papers No. 2008 - 037). Jena: Friedrich Schiller University and Max Planck Institute of Economics.

Wilson, A. G. (1967). A Statistical Theory of Spatial Distribution Models. Transportation Research, 1, 253-69.

Wilson, A. G. (1970). Entropy in Urban and Regional Modelling. London: Pion.

Witt, S. F. and Witt, C. A. (1995). Forecasting Tourism Demand: A Review of Empirical Research. International Journal of Forecasting, 11(3), 447-75.

Yang, C.-H., Lin, H.-L. and Han, C.-C. (2010). Analysis of International Tourist Arrivals in China: The Role of World Heritage Sites. Tourism Management, 31(6), 827-37.

Zhang, J. and Jensen, C. (2007). Comparative Advantage: Explaining Tourism Flows. Annals of Tourism Research, 34(1), 223-43. 


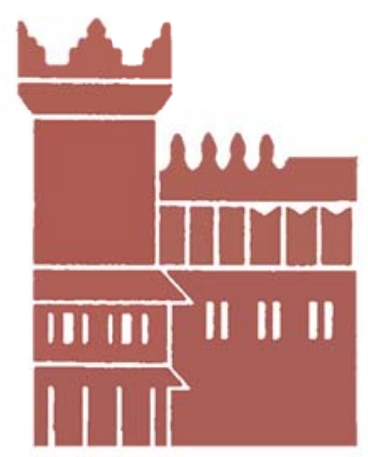

Alma Mater Studiorum - Università di Bologna DEPARTMENT OF ECONOMICS

Strada Maggiore 45

40125 Bologna - Italy

Tel. +39051 2092604

Fax +390512092664

http://www.dse.unibo.it 